14

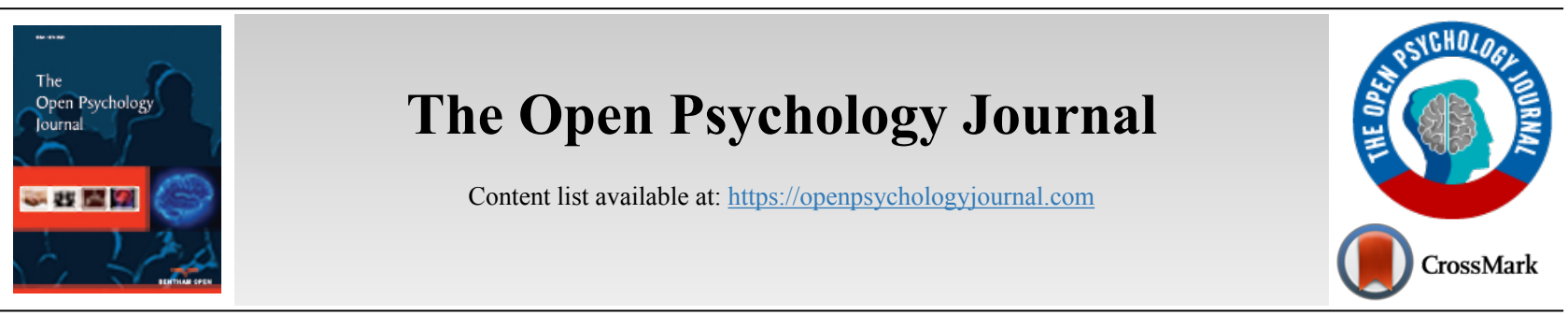

EDITORIAL

\title{
Use of Artificial Intelligence to Assess Human Emotion
}

\author{
Thiago Gonçalves dos Santos Martins ${ }^{1,2,3, *}$, Rufino Silva ${ }^{2,4}$, Luís Guilherme Arneiro Mendes ${ }^{4}$ and Paulo Schor ${ }^{1}$ \\ ${ }^{1}$ Federal University of São Paulo (UNIFESP), Sao Paulo, Brazil \\ ${ }^{2}$ University of Coimbra, Coimbra, Portugal \\ ${ }^{3}$ Ludwig Maximilians Universitat (LMU), Munich, Germany \\ ${ }^{4}$ AIBILI (Association for Biomedical Research in Light and Image), Coimbra, Portugal
}

Keywords: Health professionals, Patients relations, Health education, Artificial intelligence, Human emotion, Hippocratic medicine.

\section{INTRODUCTION}

The relationship of health professionals with patients emerged together with Hippocratic medicine, whose goal was to treat the person and not just focus on the disease [1]. The relationship of health professionals with patient is fundamental in promoting the quality of care. The short time for consultations and the use of technology have been pointed as factors that hinder empathy, making a relationship of health professional with patient as a mere search for symptoms for the prescription of drugs.[2]. The development of biochemistry, pharmacology, immunology, and genetics also contributed to the growth of a disease-centered biomedical model, thereby diminishing interest in the patient's experience. The new more sophisticated techniques have played an important role in diagnosis rather than the personal relationship between health professionals and patients. Technology has been incorporated into the profession, leaving aside the subjective aspect of the relationship. The place of the individual became the bearer of lesions, which are viewed with interest and positivity by the health professional [3].

From Donabedian's studies in the 1970s and 1980s, it is known that the quality of health services, as perceived by patients, depends on 30 to $40 \%$ of the physician's diagnostic and therapeutic capacity, and 40 to $50 \%$ on the relationship established between health professionals and users [3].

Increasingly shorter consultations often interfere with the patient- health professional relationship, making it difficult to establish empathy. This refers to the health professional 's awareness of the changes felt and reflected by the patient. This

\footnotetext{
* Address correspondence to this author at Federal University of São Paulo and University of Coimbra Botucatu st, 821 Vila Clementino, São Paulo Postal, code: 04023-062, Sao Paulo, Brazil; Tel: +552125712248;

E-mail thiagogsmartins@yahoo.com.br.
}

relationship is not only important for patient satisfaction, but also adherence to treatment. The importance of the health professional is used in Ambroise Paré's phrase, which evokes "heal occasionally, often relieve, always comfort". However, health professional ends up taking refuge in specialties, referring patients to various specialists, who in lightning consultations do not take into consideration the patient as a whole biopsychosocial.

In 2009 , it was found that $32 \%$ of medical errors in the United States resulted from decreased consultation times, producing misdiagnosis and worsening patient outcomes. Even in hospitals that have electronic medical records, with the possibility of better data collection, it is admitted that $78.9 \%$ of medical errors would be related to problems in the doctorpatient relationship and failure to evaluate patient data [4].

It is noticed that health professional education has not prioritized the approach to issues related to the interaction between health professionals and patients. The focus on communication skills is fundamental.

The analysis of various postures and attitudes allows the student to build a critical view of the relationship with patients.

The patient's health-professional relationship is influenced by the psychosocial aspects of the patient with his illness, his expectations, fears, anxieties, previous experiences of other health professionals, as well as, by the professionals themselves, with his personality, his psychological factors (stress, anxiety, frustration) and their technical training (professional experience and communication skills).

At the same time as discussing problems in the health professional- patient relationship and the deficiency of clinical examination in health care, which makes clinical diagnosis more dependent on complementary examinations, the 
importance of the computer is increasingly emphasized. Data storage and processing capacity have increased exponentially (big data). Artificial intelligence processes this data through algorithms, which tend to be refined by their operation and to propose increasingly accurate diagnostic hypotheses. This technology that can contribute to the worsening relationship with patients can also be used to improve the quality of health education by developing listening, examining and guiding a patient. It is well known that a person's emotional state can influence their facial movements and tone of voice and this information is important for the interaction between people.

To improve the communication skills of health professionals with patients, there are algorithms using artificial intelligence programs that analyze the emotion present in the face and voice of the health professional. The assessment of the emotional state that health professionals are in consultations can facilitate the development of empathy with patients. We can try to use the technology that has been pointed out in several studies as responsible for the worsening health professional patient relationship, allied in the teaching of health professionals.

Artificial intelligence began in 1950 when Alan Turing published his article "Computing Machinery and Intelligence" and proposed a test that compares the performance of a computer and a person in solving a problem. The term artificial intelligence was established at a conference at Dartmouth College in 1956 by McCarthy et al., And its application in medicine began with Shortlife's article in 1963 [5].

\subsection{Facial Emotions Recognition Model}

In the nineteenth century, Darwin began the study of facial and muscle expression that accompany various emotions. He suggested that the expression of emotion would be a trace of reactions in ancestral species. Emotions are adaptations that integrate the mechanisms by which organisms regulate life. Emotions lead to creating favorable circumstances for the organism in which the phenomenon manifests itself. This way your understanding can be useful to improve the relationship between health professionals and patients.

Emotion recognition algorithms have already been developed by analyzing people's faces. Microsoft has an algorithm that uses machine learning to quantify feelings such as anger, fear, happiness, sadness, surprise, and neutrality of feelings. This happens with the presentation of a large database with thousands of images classified with their feelings. The more images are presented to the algorithm, the greater the chance of success when classifying a new image.

Pattern recognition that is naturally learned by humans is being enhanced by artificial intelligence techniques. They try to represent the pattern as a numeric vector, called a similarity vector. The pattern will be classified according to the greatest similarity between it and the representative vector. This can be achieved by neural networks that adjust to new information, providing good answers even with missing or confusing data. The ability to generalize the neural network presents an advantage in the problem of pattern recognition never seen before, but similar to those presented during training.
Face-recognition algorithms have an accuracy of approximately $90 \%$, but for emotion recognition, this accuracy is reduced to $70 \%$, yet it is still far from the human capacity that has been developed since we were born [6].

The study of facial muscle movements is important for the study of emotions. Several companies say they have already been able to detect emotions on the faces of people like Microsoft's Affectiva. For example, algorithms identify some characteristics of people's face position as characteristic of feelings. As an example, we have pride, which can be interpreted with acts of lifting the head and eyes down or feelings of surprise in which one would present; Eyebrows raised, upper eyelid raised, lips parted, jaw dropped.

However, humans have about 34 muscles responsible for facial expressions, which contract and relax to express various emotions, demonstrating the complexity of correctly capturing these feelings in addition to the anatomical variations between various people [7].

However, despite all the technological advancement it is still difficult to pinpoint emotion on people's faces, as there is great variability of facial movements between people for the same emotion that has not yet been learned by algorithms.

\subsection{Voice Emotion Recognition Model}

Some algorithms have up to $64 \%$ accuracy in recognizing feelings by voice [8].

Voice recognition begins at the sound pickup stage and turns it into a digital signal. In the preprocessing phase, there is the formation of a characteristic vector of the pattern to be analyzed and the elimination of redundant signals. In the processing phase, there is pattern recognition. Voice recognition of emotions using neural network techniques is effective.

The use of two emotion analysis algorithms increases the final accuracy in detecting the health professional's emotional state. This allows feedback on how their emotions are manifesting and a possible improvement in empathy and patient relationship, allowing for improved outcomes and adherence to treatment, increasing the degree of patient satisfaction.

The algorithms presented are not the most accurate for measuring human emotion, as they depend on subjective criteria. Objective emotion measurement techniques may be more accurate. These can be noticed with changes in the autonomic nervous system, such as the cardiovascular, respiratory system and variations in skin conductance. These measurements have numbers and do not need subjective human interpretation to present the results.

However, current algorithms still have limitations, as their database classifications are performed on stereotyped emotion classifications and require a larger database with various ethnic groups. The form of feeling expression is still very variable across cultures. and people and a facial movement can represent more than one emotional category. Even some humans have difficulty categorizing emotions because of their wide variations. New research in the area of emotion should 
consider samples from individuals in different situations and times of the day. This large database considering not only facial movements but also a tone of voice can provide better "fingerprints" of emotions for future studies, improving the perception of feelings and can be applied in improving the relationship between health professionals and patients.

The technology that is pointed out in many studies by one of the main contributors to the decrease of health professionals' empathy for patients can be used as an ally in the development of algorithms to improve the health professional-patient relationship. The biggest profit from this change is to have health professionals who are better able to take care of the health of patients in their community. A better health professional-patient relationship not only has positive effects on user satisfaction and quality of health services but also has a direct influence on patients' health status.

\section{REFERENCES}

[1] Daikos KG. History of medicine: Our hippocratic heritage. Int J Antimicrob 2007; 29: 617-20.

[2] Smith R. Why are doctors so unhappy? There are probably many causes, some of them deep. BMJ 2001 n; 322(7294): 1073-4. [http://dx.doi.org/10.1136/bmj.322.7294.1073] [PMID: 11337419]

[3] Donabedian A. La qualita dell'assistenza sanitaria. Roma: NIS 1990.

[4] Castaneda C, Nalley K, Mannion C, et al. Clinical decision support systems for improving diagnostic accuracy and achieving precision medicine. J Clin Bioinforma 2015; 5(4): 4.

[http://dx.doi.org/10.1186/s13336-015-0019-3] [PMID: 25834725]

[5] Shortliffe EH. The adolescence of AI in medicine: will the field come of age in the '90s. Artif Intell Med 1993; 5(2): 93-106. [http://dx.doi.org/10.1016/0933-3657(93)90011-Q] [PMID: 8358494]

[6] Arcaro MJ, Schade PF, Vincent JL, Ponce CR, Livingstone MS Seeing faces is necessary for face-domain formation. Nat Neurosci 2017; 20(10): 1404-12.

[http://dx.doi.org/10.1038/nn.4635] [PMID: 28869581]

[7] Neth D, Martinez AM. Emotion perception in emotionless face images suggests a norm-based representation. J Vis 2009; 9(1): 1-11. [http://dx.doi.org/10.1167/9.1.5] [PMID: 19271875]

[8] Jiang W, Wang Z, Jin JS, Han X, Li C. 2019; Speech emotion recognition with heterogeneous feature unification of deep neural network. Sensors (Basel) 18;19(12)

[http://dx.doi.org/10.3390/s19122730]

(C) 2020 dos Santos Martins et al.

This is an open access article distributed under the terms of the Creative Commons Attribution 4.0 International Public License (CC-BY 4.0), a copy of which is available at: https://creativecommons.org/licenses/by/4.0/legalcode. This license permits unrestricted use, distribution, and reproduction in any medium, provided the original author and source are credited. 